Ion acoustic wave damping in a non-Maxwellian bi-ion electron plasma in the presence of dust

Kashif Arshad, Yana G. Maneva, and S. Poedts

Citation: Physics of Plasmas 24, 093708 (2017); doi: 10.1063/1.4995581

View online: http://dx.doi.org/10.1063/1.4995581

View Table of Contents: http://aip.scitation.org/toc/php/24/9

Published by the American Institute of Physics

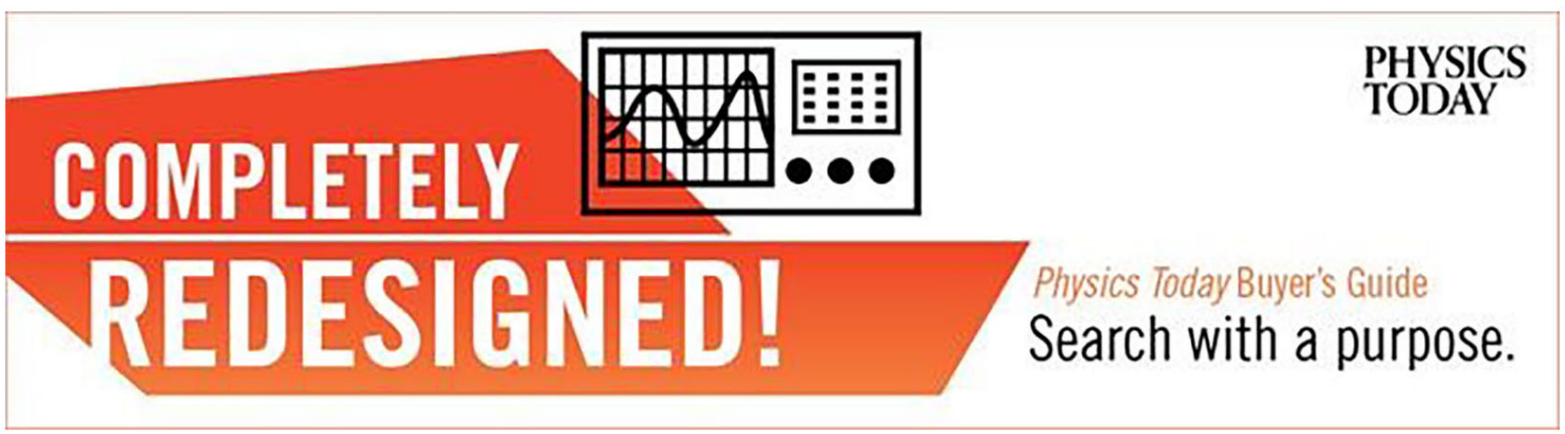




\title{
Ion acoustic wave damping in a non-Maxwellian bi-ion electron plasma in the presence of dust
}

\author{
Kashif Arshad, ${ }^{1,2, a)}$ Yana G. Maneva, ${ }^{1, b)}$ and S. Poedts ${ }^{1}$ \\ ${ }^{1}$ Centre for Mathematical Plasma Astrophysics, KU Leuven, Celestijnenlaan 200B, 3001 Leuven, Belgium \\ ${ }^{2}$ Pakistan Institute of Engineering and Applied Sciences, P. O. Nilore, Islamabad 45650, Pakistan
}

(Received 12 July 2017; accepted 7 September 2017; published online 29 September 2017)

In this work, we investigate the properties of ion acoustic waves generated by negative heavy ions in a multi-component non-Maxwellian electrostatic plasma, consisting of electrons, positive and negative heavy ions, and a background population of dust particles. In the absence of external energy source and collisions, the ion acoustic waves are subject to Landau damping. We perform a collisionless linear instability analysis within a slow damping approximation and study the effect of various plasma conditions on the damping rate. Our model is highly relevant to multi-ion laboratory plasmas and has been applied to $\mathrm{K}^{+}-\mathrm{SF}_{6}^{-}$and $\mathrm{K}^{+}-\mathrm{C}_{7} \mathrm{~F}_{14}^{-}$mixtures in particular. Published by AIP Publishing. [http://dx.doi.org/10.1063/1.4995581]

\section{INTRODUCTION}

An ion acoustic wave (IAW) is an electron temperature driven wave which involves density fluctuations owing to the longitudinal ion and electron oscillations under the influence of electromagnetic fields and particle collisions. ${ }^{1}$ Being first predicted by Tonks and Langmuir ${ }^{2}$ and demonstrated by Revans in a gas discharge tube, ${ }^{3}$ the phase velocity of IAW was predicted by Wong et al. ${ }^{4}$ to be much smaller than the electron thermal velocity in a Q-machine plasma, but much greater than the ion thermal velocity, with the pressure provided by the electrons and the inertia provided by the ions. In the absence of external forces, the IAWs are attenuated by both Coulomb collisions and collisionless Landau damping, unless the electron temperature $T_{e}$ is much higher than the ion temperature $T_{i}$ when the IAWs can become unstable. Landau damping ${ }^{59}$ is one of the most famous fundamental kinetic phenomena in classical plasma physics, which takes into account the effects of the velocity space configuration. If the phase velocity of the excited IAW is approximately equal to the thermal speed of ions or electrons, a resonant wave-particle interaction called Landau resonance (or Landau damping) occurs. When this happens, the suprathermal particles at the tail of the velocity distribution function can extract energy from the faster wave and damp it. In other words, significant Landau damping occurs when the phase velocity of the IAW approaches the ion thermal velocity or the electrons' thermal velocity. This wave-particle interaction leads to net transfer of wave energy to the slower particles eventually causing the wave to be damped. The ion Landau damping in collisionless plasmas is established to be strong when $T_{i}$ approaches $T_{e}$, and in the absence of particle collisions, the IAW can only propagate without noticeable attenuation if $T_{e} \gg T_{i}$. The contribution of singularities $v=$ $v_{p h} \equiv \omega / k$ is vital for the collisionless damping process, where the particles with velocity $v$ are in resonance with the phase speed of the IAW $v_{p h}$.

\footnotetext{
a)kashif.arshad.butt@gmail.com and kashif.arshad@kuleuven.be

b)yana.maneva@kuleuven.be
}

The presence of heavy charged species of variable sizes is very common in space and astrophysical plasmas, which often consist of complex multi-component dusty plasma. Dusty plasmas prevalently exist in various space regions ${ }^{5,6}$ and astrophysical environments such as interstellar and circumstellar clouds, ${ }^{7,8}$ as well as in interplanetary space and planetary rings, such as the spokes in Saturn rings ${ }^{9-11}$ and the Earth's ionosphere. ${ }^{12}$ These plasmas are admixture of ionized gases and charged microscale dust particles with various interdisciplinary applications and perspectives. ${ }^{13-15}$ Similar to IAWs, it is well known that two normal modes existing in dusty plasmas are the dust acoustic (DA) and dust ion acoustic (DIA) waves. ${ }^{16}$ DA waves are attributed to the dust dynamics, whereas for DIA waves, the wave phase velocity is larger than both the dust and ion thermal velocities. In the case of DIA waves, the inertia is provided by the dust and ions while the restoring force comes from the electron pressure. When the restoring pressure is exerted by inertia-less electrons and ions while the massive dust particles provide the inertia, the excited fluctuations are called DA waves.

There has been increasing interest in research on low frequency dynamics in dusty plasmas nowadays. Recently, DA rogue waves have been studied in a non-thermal plasma in Ref. 17, where the velocity distributions deviate from Maxwellian. Later, a non-linear approach to study the propagation of DIA waves in different geometries has been applied in Ref. 18. Previously, the dust confinement and existence of dust acoustic waves in anodic magneto active plasmas have been predicted in Ref. 19. The presence of multi-ion dusty plasmas in laboratory and space and their theoretical and laboratory aspects have been studied in great detail by the authors of Refs. 20-26 and collaborators. These authors have revealed various properties of IAWs, including the related plasma instabilities and relevant parameters in multi-ion plasmas. Cometary dusty plasmas are more complicated and potentially unstable where many types of ions are present. ${ }^{27} \mathrm{Kim}$ and Merlino ${ }^{28}$ have studied the electron attachment to perfluoromethylcyclohexane $C_{7} F_{14}$ and sulfur 
hexafluoride $S F_{6}$ in a $Q$ machine which in turn produces a thermally ionized plasma containing negative ions $S F_{6}^{-}$and $C_{7} F_{14}^{-}$. The generation of a dust negative ion acoustic wave (DNIAW) in two plasmas composed of electrons, positively charged $K^{+}$ions, and negatively charged $S F_{6}^{-}$and $C_{7} F_{14}^{-}$ ions with immobile dust particles has been reported in Ref. 29. Also, the damping rates and frequency shifts of IAWs in multi-ion plasmas have been studied in Ref. 30. Most of the existing works related to the theoretical formulation and experimental observations of IAWs in multi-component dust plasmas are based on the classical fluid theory and kinetic modeling with Maxwellian velocity distributions. ${ }^{31-33}$ Yet, these plasmas are susceptible to various effects, for example, the presence of energetic particles, spatial changes in the plasma properties, and external force fields, which can lead to more complicated velocity configurations. The presence of energetic particles for instance gives rise to the Lorentzian or kappa $(\kappa)$ distribution function, ${ }^{34}$ which is the focus of the present study.

Over the last few decades, the kinetic study of IAWs has taken the plasma physics into a new era. ${ }^{35} \mathrm{~A}$ lot of manuscripts have been based on the propagation of waves in thermally distributed Maxwellian plasmas. Yet, it is observed that space plasmas are best described by the non-thermal distributions. ${ }^{36-39}$ This leads to the non-thermally distributed nonlinear study of ion acoustic modes ${ }^{40,41}$ in the absence and in the presence of dust grains. ${ }^{42}$ The further modified ion acoustic modes are extracted by the two-temperature (Boltzmann and kappa distributed) electrons. ${ }^{43-47}$ When a streaming plasma beam permits a fixed target, a kinetic instability is generated within the target plasma ${ }^{27}$ due to the energy transfer from the source (streaming plasma). In recent years, it has been shown that the non-thermal (non-Maxwellian) distributions ${ }^{48,49}$ are frequently observed in space and most suitable for instability studies in dusty and multi-ion plasmas. ${ }^{50,51}$

Here, our aim is to theoretically investigate the propagation of IAWs and their collisionless damping in kappadistributed bi-ion electron plasmas (e.g., $K^{+}-S F_{6}^{-}$and $K^{+}-C_{7} F_{14}^{-}$) in the presence of stationary background negatively charged dust particles. We perform linear instability analysis and study the variation of the Landau damping rate as a function of various plasma properties. We highlight the influence of the electron-to-negative ion temperature ratio, as well as the $\kappa$ index of the non-thermal velocity distributions and the charging of the background dust population. The paper is organized as follows: in Sec. II, the model description is provided and the wave dispersion properties are obtained analytically with the derivation of the damping rate and the propagation frequency. Section III is devoted to numerical results and discussion with a summary of the results at the end.

\section{DISPERSION AND DAMPING OF IAWS IN A KAPPA- DISTRIBUTED DUST BI-ION ELECTRON PLASMA}

Let us consider a non-Maxwellian multi-species plasma composed of electrons, a lighter population of positive ions (e.g., Potassium: $K^{+}$), a heavy population of negative ions (e.g., sulfur hexafluoride: $S F_{6}^{-}$or perfluoromethylcyclohexane:
$C_{7} F_{14}^{-}$), and negatively charged background dust grains. The charge neutrality condition for such dust-multi-ion-electron (DBIE) plasma is given by

$$
Z_{+} n_{0+}=n_{0 e}+Z_{-} n_{0-}+Z_{d} n_{0_{d}},
$$

where $n_{0+}, n_{0 e}, n_{0-}$, and $n_{0_{d}}$ are the background number densities of the positive ions, electrons, negative ions, and dust grains, respectively. $Z_{+}$and $Z_{-}$are the positive and negative ion states and $Z_{d}$ is the charging parameter of the dust grains. In the absence of collisions, the dynamics of each plasma species $\alpha$ is determined by the Vlasov equation

$$
\frac{\partial f_{\alpha}}{\partial t}+\mathbf{v} \cdot \nabla f_{\alpha}+\mathbf{a} \cdot \nabla_{v} f_{\alpha}=0
$$

where the acceleration is given by $\mathbf{a}=q_{\alpha}(\mathbf{E}+\mathbf{v} \times \mathbf{B}) / m_{\alpha}$ and $\alpha=e,+,-, d$ for the electrons, positive ions, negative ions, and dust grains, respectively, with $q_{\alpha}$ and $m_{\alpha}$ being the corresponding charge and mass. We assume that there are no macroscopic fields acting on the background system, $\mathbf{E}_{0}=0$ and $\mathbf{B}_{0}=0$. In this study, we treat the examined DBIE plasma as unmagnetized or consider only longitudinal oscillations which arise from motions parallel to the magnetic field when the $\mathbf{B}$ field is present. As a first attempt to study the effects of non-thermal kappa distributions on the dynamics of the plasma species and the propagation of IAWs, we base our model on the assumption of a fixed charge of the dust grains, which does not fluctuate. For more realistic conditions, the dust charge can be perturbed and inelastic collisions (related to charging of dust grain and capture of plasma particles) can play an important role and yield considerably different results. These effects have been studied in detail in Ref. 52 and other references cited therein. In the limit of large wave-lengths, the damping rate due to dust charging can become several orders of magnitude larger than the Landau damping caused by kinetic effects, while in the short wave-length domain, the charging effects are overcome by the non-thermal dynamics and can be neglected. The collision rate also becomes negligible and may be ignored provided that the number density of the dust particle is very low. In this article, we will neglect the effect of the collisions and will base our model on a fixed charge of the dust grains (as previously reported in several references within Ref. 53). When we perturb and Fourier transform the Vlasov equation (1), the perturbed linear distribution function in the case of longitudinal electrostatic fluctuations becomes

$$
f_{\alpha 1}=-\frac{q_{\alpha}}{i m_{\alpha}} \frac{\mathbf{E}_{1} \cdot \nabla_{v} f_{\alpha 0}}{\left(\omega-k v_{z}\right)} .
$$

The plasma current is given by the standard expression $\mathbf{J}_{\alpha}$ $=\sum_{\alpha} q_{\alpha} n_{\alpha} \mathbf{v}_{\alpha}$ which can be used to calculate the conductivity tensor. With the help of the conductivity tensor, we can calculate the dielectric tensor. The macroscopic density and velocity for the $\alpha$ species are defined as the zeroth and the first moment of the distribution function $n_{\alpha}=\int f_{\alpha} d^{3} \mathbf{v}$ and $\mathbf{v}_{\alpha}=\left(\int \mathbf{v} f_{\alpha} d^{3} \mathbf{v}\right) / n_{\alpha}$, respectively. We may now substitute the definitions of $n_{\alpha}, \mathbf{v}_{\alpha}$, and $f_{\alpha}$ in the expression for the plasma current density defined in the linear limit $J_{r 1}=\sigma_{r s} E_{s 1}$, where 
$\sigma_{r s}$ are the components of the conductivity tensor and the summation is over the repeated index $s$. Following this standard procedure for the components of the conductivity tensor, we obtain

$$
\sigma_{r s}=-\sum_{\alpha} \frac{q_{\alpha}^{2}}{i m_{\alpha}} \iiint \frac{\mathbf{v}_{r} \partial f_{\alpha 0} / \partial v_{s}}{\left(\omega-k v_{z}\right)} d^{3} \mathbf{v}
$$

As a next step, we choose a non-Maxwellian equilibrium velocity distribution function, such as $\kappa$ or generalized Lorentzian distribution ${ }^{34,40}$

$$
f_{\alpha 0}=\frac{1}{\pi^{3 / 2} \theta_{\perp \alpha}^{2} \theta_{\| \alpha}} \frac{\Gamma(\kappa+1)}{\kappa^{3 / 2} \Gamma\left(\kappa-\frac{1}{2}\right)}\left[1+\frac{v_{\|}^{2}}{\kappa \theta_{\| \alpha}^{2}}+\frac{v_{\perp}^{2}}{\kappa \theta_{\perp \alpha}^{2}}\right]^{-\kappa-1},
$$

where $\kappa$ is the Lorentzian index with values $\kappa>3 / 2$. The Lorentzian thermal speed is related to the particle temperature by the relation, $\theta_{\| \alpha, \perp \alpha}^{2}=\left(\frac{2 \kappa-3}{\kappa}\right) v_{T_{\| \alpha}, \perp \alpha}^{2}$ and the thermal velocity of the $\alpha$ species is defined as $v_{T_{\| \alpha, \perp \alpha}}=\sqrt{T_{\| \alpha, \perp \alpha} / m_{\alpha}}$. The spectral index $\kappa$ is a measure of the slope of the energy spectrum of the supra-thermal particles in the tail of the velocity distribution function. ${ }^{21}$ The smaller the value of $\kappa$, the higher the number of supra-thermal particles in the tail of the distribution function and the harder the energy spectrum. As $\kappa \rightarrow \infty$, the non-thermal kappa distribution approaches a thermal Maxwellian.

After using the Kappa distribution function described in Eq. (4) in Eq. (3), the conductivity tensor is obtained as follows:

$$
\begin{aligned}
\sigma_{r s}= & -\frac{i \epsilon_{0}}{\pi^{3 / 2}} \sum_{\alpha} \frac{\omega_{p \alpha}^{2}}{\theta_{\perp \alpha}^{2} \theta_{\| \alpha}^{3}} \frac{\Gamma(\kappa+1)}{\kappa^{3 / 2} \Gamma\left(\kappa-\frac{1}{2}\right)} \frac{(\kappa+1)}{\kappa} \\
& \times \int \frac{v_{r} v_{s}}{\left(\omega-k v_{\|}\right)}\left[1+\frac{v_{\|}^{2}}{\kappa \theta_{\| \alpha}^{2}}+\frac{v_{\perp}^{2}}{\kappa \theta_{\perp \alpha}^{2}}\right]^{-\kappa-2} d^{3} \mathbf{v}
\end{aligned}
$$

where $\omega_{p \alpha}=\sqrt{4 \pi n_{0 \alpha} q_{\alpha}^{2} / m_{\alpha}}$ is the plasma frequency of the $\alpha$ species and $r$ and $s$ in the subscript denote the coordinate axes of the given coordinate system. Now performing perpendicular integration on the integral in Eq. (5), the dielectric function is obtained as follows:

$$
\epsilon_{r s}=\delta_{r s}+\sum_{\alpha} \frac{\omega_{p \alpha}^{2}}{2 k^{2} \theta_{\| \alpha}^{2}}[1+\xi Z(\xi)]
$$

where $\epsilon_{r s}=\delta_{r s}+i \sigma_{r s} /\left(\omega \epsilon_{0}\right)$ has been defined and $\xi=\omega / k \theta_{\| \alpha}$. It can be seen that as the non-thermal index $\kappa \rightarrow \infty$, the Lorentzian thermal velocity approaches the ordinary thermal velocity and the above dielectric function equation (6) is transformed into the usual Maxwellian distributed dielectric function. The dispersion function $Z\left(\omega / k \theta_{\| \alpha}\right)$ for the non-Maxwellian plasmas is defined as

$$
Z\left(\frac{\omega}{k \theta_{\| \alpha}}\right)=\frac{1}{\sqrt{2 \pi}} \int_{-\infty}^{\infty} \frac{v_{r} v_{s}}{\left(\omega-k v_{\|}\right)}\left[1+\frac{v_{\|}^{2}}{\kappa \theta_{\| \alpha}^{2}}\right]^{-\kappa-1} d \mathbf{v}_{\|} .
$$

The above defined dispersion function can be expanded in the limits of large and small ratios $\left|\omega / k \theta_{\| \alpha}\right|$. For the longitudinal modes, we will take $v_{\|}=v_{r}=v_{s}$. So the large $\left|\omega / k \theta_{\| \alpha}\right| \ll 1$

$$
Z\left(\frac{\omega}{k \theta_{\| \alpha}}\right)=-i \sqrt{\pi}\left(\frac{\omega}{k \theta_{\| \alpha}}\right) \frac{\Gamma(\kappa+1)}{\kappa^{3 / 2} \Gamma\left(\kappa-\frac{1}{2}\right)}\left[1+\frac{\omega^{2}}{\kappa k^{2} \theta_{\| \alpha}^{2}}\right]^{-\kappa-1},
$$

and $\left|\omega / k \theta_{\| \alpha}\right| \gg 1$, we have

$$
\begin{aligned}
Z\left(\frac{\omega}{k \theta_{\| \alpha}}\right)= & +\left(\frac{k \theta_{\| \alpha}}{\omega}\right)^{2}+\frac{3 \kappa}{2(3 \kappa-2)}\left(\frac{k \theta_{\| \alpha}}{\omega}\right)^{4} \\
& +\mathcal{O}\left(\frac{k \theta_{\| \alpha}}{\omega}\right)^{6}-i \sqrt{\pi}\left(\frac{\omega}{k \theta_{\| \alpha}}\right) \frac{\Gamma(\kappa+1)}{\kappa^{3 / 2} \Gamma\left(\kappa-\frac{1}{2}\right)} \\
& \times\left[1+\frac{\omega^{2}}{\kappa k^{2} \theta_{\| \alpha}^{2}}\right]^{-\kappa-1} .
\end{aligned}
$$

In the limit $\kappa \rightarrow \infty$, the real part of the above expressions of the plasma dispersion function corresponds to the real part of the famous Fried Conte Function. ${ }^{54}$ The ordering of phase velocity $v_{\phi}=\omega / k$ and Lorentzian thermal velocities of the respective mode can be written as

$$
\theta_{\|_{-}} \ll v_{\phi} \ll \theta_{\|_{e}}, \theta_{\|_{+}} .
$$

This suggests that the inertia of heavy negative ion is important in comparison to positive ions and electrons while the dust grains are stationary. The longitudinal ion acoustic wave in the DBIE plasmas can be obtained by the expansion of the dielectric function

$$
\begin{aligned}
\epsilon_{\| \alpha}= & +\frac{\omega_{p e}^{2}}{2 k^{2} \theta_{\| e}^{2}}+\frac{\omega_{p+}^{2}}{2 k^{2} \theta_{\|+}^{2}}-\frac{\omega_{p-}^{2}}{2 \omega^{2}}\left[1+\frac{3 \kappa}{2(3 \kappa-2)} \frac{k^{2} \theta_{\|-}^{2}}{\omega^{2}}\right] \\
& +i \sqrt{\pi} \frac{\omega}{k^{3}} \frac{\Gamma(\kappa+1)}{\kappa^{3 / 2} \Gamma\left(\kappa-\frac{1}{2}\right)}\left[\frac{\omega_{p e}^{2}}{\theta_{\| e}^{3}}+\frac{\omega_{p+}^{2}}{\theta_{\|+}^{3}}\right. \\
& \left.+\frac{\omega_{p-}^{2}}{\theta_{\|-}^{3}}\left(1+\frac{\omega^{2}}{\kappa k^{2} \theta_{\|-}^{2}}\right)^{-\kappa-1}\right] .
\end{aligned}
$$

The angular frequency is described as $\omega=\omega_{r}+i \gamma$ for the slowly growing and decreasing mode with the assumption that the imaginary part of the dispersion relation is much smaller than the real part, i.e., $\left|\omega_{r}\right| \gg|\gamma|$. The dispersion relation for the ion acoustic wave for a Kappa distributed (or Lorentzian) DBIE plasmas is described as follows:

$$
\omega_{r}^{2}=\frac{k^{2} \omega_{p-}^{2} \lambda_{D}^{2}}{\left(\frac{\kappa}{2 \kappa-3}+2 k^{2} \lambda_{D}^{2}\right)}\left[1+\frac{3 \kappa}{2(3 \kappa-2)} \frac{k^{2} \theta_{\|-}^{2}}{\omega^{2}}\right],
$$

where the effective Debye length of the species is defined as $\lambda_{D}=\left[\lambda_{D e}^{-2}+\lambda_{D+}^{-2}\right]^{-1 / 2}$. In the absence of positive ions $\left(n_{0+}=0\right.$, which implies $\left.\lambda_{D+}^{-2}=0\right)$ and by replacing the subscript of the negative ions by the general one " $i$ " for the ions, the dispersion relation becomes 


$$
\omega_{r}^{2}=\frac{k^{2} \omega_{p i}^{2} \lambda_{D e}^{2}}{\left(\frac{\kappa}{2 \kappa-3}+2 k^{2} \lambda_{D e}^{2}\right)}\left[1+\frac{3 \kappa}{2(3 \kappa-2)} \frac{k^{2} \theta_{\|_{i}}^{2}}{\omega^{2}}\right] .
$$

In the larger limit of the spectral index such that $\kappa \rightarrow \infty$, the above form of the dispersion relation is analogous to the one described for the Maxwellian distributed plasma in Refs. 1 and 54.

The damping rate $\gamma$ of the ion acoustic wave in DBIE plasma is obtained by using the definition $\gamma=-\epsilon_{i} /$ $\left(\partial \epsilon_{r} / \partial \omega_{r}\right)$, which is given by

$$
\begin{aligned}
\gamma= & -\sqrt{\frac{\pi}{8}} \frac{\Gamma(\kappa+1)}{(\kappa-3 / 2)^{3 / 2} \Gamma\left(\kappa-\frac{1}{2}\right)} \\
& \times \frac{k \lambda_{D} \omega_{p}^{2} \lambda_{D}^{3}}{\left(\frac{\kappa}{2 \kappa-3}+2 k^{2} \lambda_{D}^{2}\right)^{2}}\left[\frac{1}{\omega_{p e} \lambda_{D e}^{3}}+\frac{1}{\omega_{p+} \lambda_{D+}^{3}}\right. \\
& \left.+\frac{1}{\omega_{p-} \lambda_{D-}^{3}}\left(1+\frac{\omega^{2}}{\kappa k^{2} \theta_{\|-}^{2}}\right)^{-\kappa-1}\right] .
\end{aligned}
$$

Again by applying $n_{0+}=0$ and replacing the subscript "-" by " $i$ " for the negative ions, we can write the expression of the damping rate in the following way:

$$
\begin{aligned}
\gamma= & -\sqrt{\frac{\pi}{8}} \frac{\Gamma(\kappa+1)}{(\kappa-3 / 2)^{3 / 2} \Gamma\left(\kappa-\frac{1}{2}\right)} \\
& \times \frac{k \lambda_{D e} \omega_{p}^{2} \lambda_{D e}^{3}}{\left(\frac{\kappa}{2 \kappa-3}+2 k^{2} \lambda_{D e}^{2}\right)^{2}}\left[\frac{1}{\omega_{p e} \lambda_{D e}^{3}}+\frac{1}{\omega_{p i} \lambda_{D i}^{3}}\left(1+\frac{\omega^{2}}{\kappa k^{2} \theta_{\|_{i}^{2}}^{2}}\right)^{-\kappa-1}\right] .
\end{aligned}
$$

We can obtain an analogous expression of damping rate from Refs. 1 and 54, applicable for the Maxwellian distributed plasmas, if the magnitude of superthermal index $\kappa$ approaches infinity. Using the value of the real frequency $\omega_{r}$ from (11), the modified form of the normalized damping rate is given by the following expression:

$$
\begin{aligned}
\frac{\gamma}{\omega_{p-}}= & -\sqrt{\frac{\pi}{8}} \frac{\Gamma(\kappa+1)}{(\kappa-3 / 2)^{3 / 2} \Gamma\left(\kappa-\frac{1}{2}\right)} \\
& \times \frac{k \lambda_{D}\left(P^{-3} Q\right)^{1 / 2}}{\left(\frac{\kappa}{2 \kappa-3}+2 k^{2} \lambda_{D}^{2}\right)^{2}}\left[1+\frac{\omega_{p e} \lambda_{D e}^{3}}{\omega_{p+} \lambda_{D+}^{3}}+\frac{\omega_{p e} \lambda_{D e}^{3}}{\omega_{p-} \lambda_{D-}^{3}}\right. \\
& \left.\times\left(1+\frac{\lambda_{D}^{2} / \lambda_{D-}^{2}}{(2 \kappa-3)\left(\frac{\kappa}{2 \kappa-3}+2 k^{2} \lambda_{D}^{2}\right)}\right)^{-\kappa-1}\right],
\end{aligned}
$$

where

$$
P=\left(2+\frac{n_{0-}}{n_{0 e}}+Z_{d} \frac{n_{0 d}}{n_{0 e}}\right), \quad Q=\frac{m_{e} n_{0-}}{m_{-} n_{0 e}} .
$$

\section{RESULTS}

In this section, we analyze the damping properties of IAWs in non-Maxwellian dust bi-ion electron (DBIE) plasma, an admixture of $K^{+}-S F_{6}^{-}$and $K^{+}-C_{7} F_{14}^{-}$, electrons, and immobile dust particles. A modified IAW in the dust bi-ion electron (DBIE) plasma is considered where the inertia of the positive light ions is neglected. We use typical parameters of DBIE plasmas obtained from the literature; ${ }^{23-26}$ the temperature of electrons and light positive ions is $T_{e}=T_{+} \simeq 0.2 \mathrm{eV}$, while for the heavy negative ions, we choose $T_{-}=0.125 T_{+}$, although the temperature ratio $T_{e} / T_{-}$can be varied. Number densities of plasma components are $n_{0 e}=10^{8} \mathrm{~cm}^{-3}$, $n_{0+}=1.46 \times 10^{9} \mathrm{~cm}^{-3}, n_{0-}=1.45 \times 10^{9} \mathrm{~cm}^{-3}, \quad$ and $n_{0 d}$ $=9 \times 10^{5} \mathrm{~cm}^{-3}$. The masses of plasma species are (electrons) $m_{e}$, (protons) $m_{p}$, (light positively charged Potassium ions) $m_{+}=39 m_{p}$, (heavy negatively charged Sulphur hexafluoride) $m_{-}=146 m_{p}$, (heavy negatively charged Perfluoromethylcyclohexane ions) $m_{-}=350 m_{p}$, and (dust grains) $m_{d}$. The dust charging parameter $Z_{d}$ for the most part of our study has a fixed value of 500 . Throughout the results, we also discuss the effect of variable number density and charging parameters for the dust particles on the dispersion properties of the damping IAWs. All damping rates as well as the real frequencies are normalized to the plasma frequency of the heavy negative ions $\gamma / \omega_{p-}$, which depends on the selected mixture.

Figure 1 shows the normalized real frequency of the modified IAWs as a function of the normalized wave numbers in the case of the $K^{+}-S F_{6}^{-}$DBIE mixture. Fig. 1(a) illustrates the dispersion as a function of the temperature ratio between the electrons and the negative ions, while Fig. 1 (b) shows the dispersion for various $\kappa$ indices. The analysis shows that the real frequency and consequently the phase speed of the IAWs decrease with increasing temperature ratios between the electrons and the Sulphur hexafluoride ions. We can also see that the real frequency decreases with the decreasing Lorentzian index. In other words, the IAWs are expected to propagate slower in highly non-thermal plasmas in comparison to thermal Maxwellian-distributed plasmas (where $\kappa \rightarrow \infty$ ).

Figure 2 plots the normalized real frequency of the modified IAWs as a function of the normalized wave numbers in the case of the $K^{+}-C_{7} F_{14}^{-}$DBIE mixture. Fig. 2(a) illustrates the dispersion as a function of the temperature ratio between the electrons and the negative ions, while Fig. 2(b) shows the dispersion for various $\kappa$ indices. The plots show the same trends as in Fig. 1 with decreasing real frequencies for increasing temperature ratios and decreasing Lorentzian indices. For a fixed range of wave numbers, the real frequency and hence the phase speed for the IAWs in the case of the heavier Perfluoromethylcyclohexane ions are several times higher than those in the case of Sulphur hexafluoride ions. Simultaneously, the dependence on the $\kappa$ index in the case of the Perfluoromethylcyclohexane ions is less prominent in comparison to the lighter Sulphur hexafluoride ions.

Figure 3 describes the normalized Landau damping rate for the light $K^{+}-S F_{6}^{-}$and heavy $K^{+}-C_{7} F_{14}^{-}$negative ion DBIE mixtures. The damping rate is shown as a function of the normalized wave number for several values 
(a)

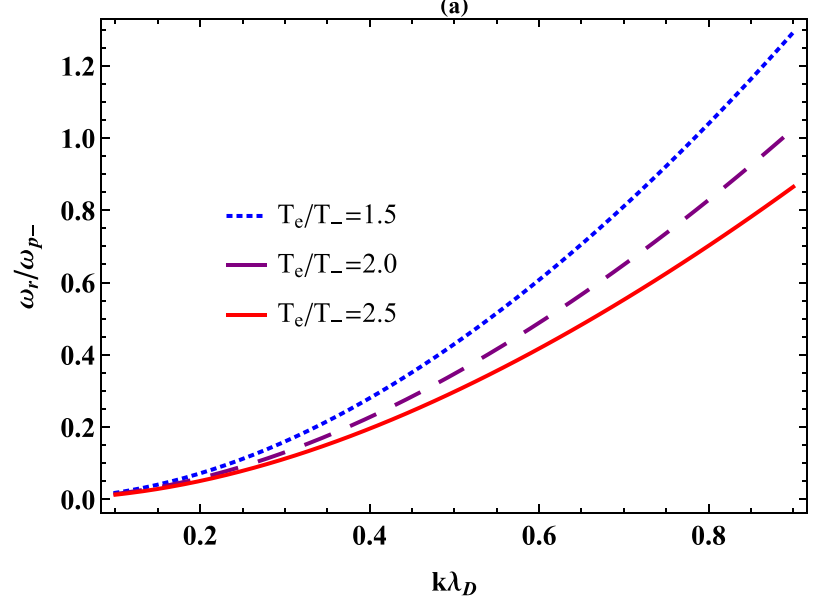

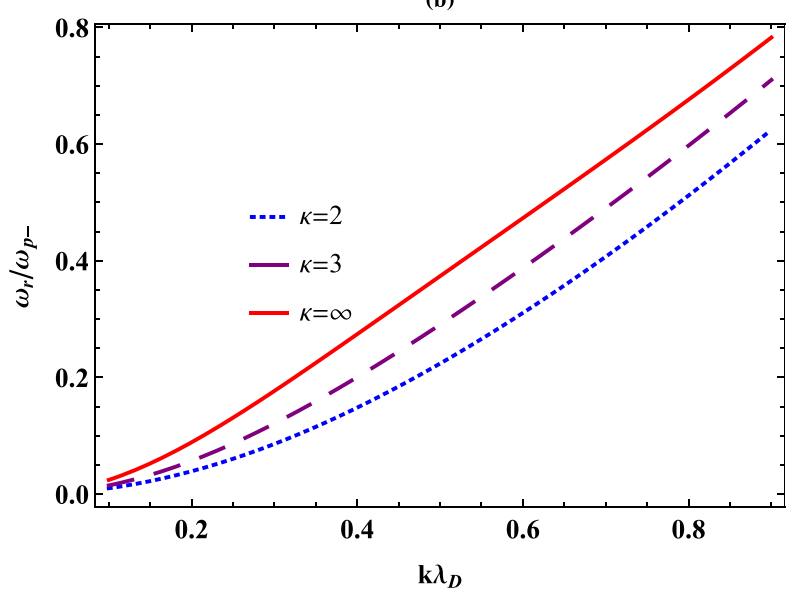

FIG. 1. The normalized real frequency of the modified IAWs in the case of the $K^{+}-S F_{6}^{-}$DBIE mixture. (a) illustrates the dispersion as a function of the temperature ratio between the electrons and the negative ions. (b) shows the dispersion $\omega / \omega_{p-}$ vs. $k \lambda_{D}$ for several spectral indices $\kappa$.
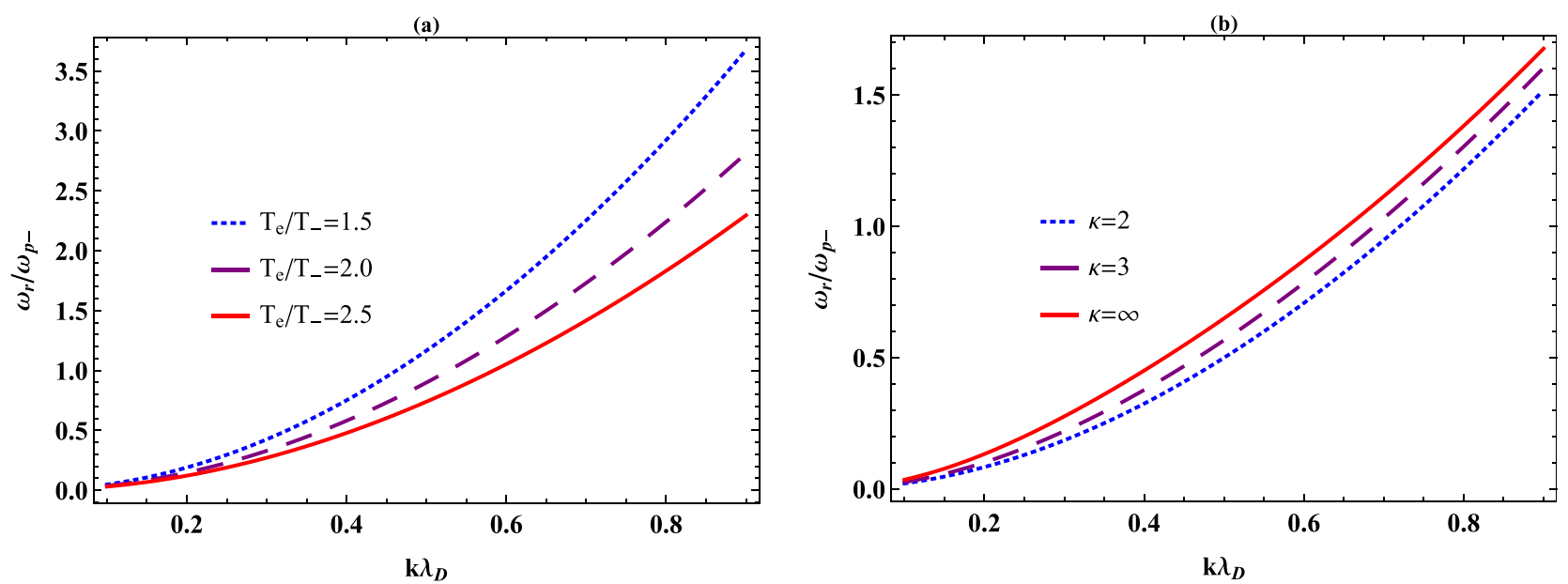

FIG. 2. The normalized real frequency of the modified IAWs in the case of the $K^{+}-C_{7} F_{14}^{-}$DBIE mixture. (a) illustrates the dispersion as a function of the temperature ratio between the electrons and the negative ions. (b) shows the dispersion $\omega / \omega_{p-}$ vs. $k \lambda_{D}$ for several spectral indices $\kappa$.

of the Lorentzian index $\kappa$. The temperature ratio of electrons-to-negative-ions for this plot is set to $T_{e} / T_{-}=2.5$. The charging potential for this case has been chosen to be $Z_{D}=500$. As expected, the Landau damping is enhanced as we decrease the values $\kappa$, as kinetic effects become

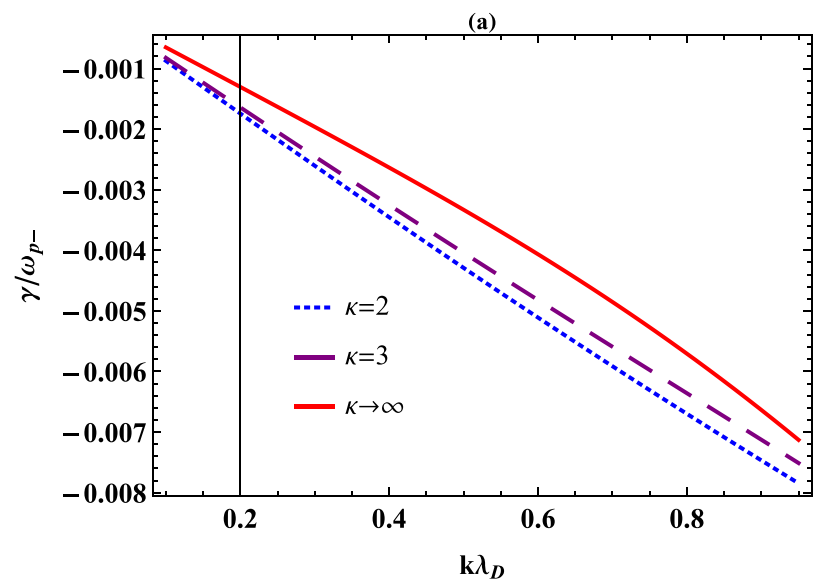

more important for highly non-thermal plasmas. The figure also shows that the damping rate for the heavier plasma (in the case of heavier negative ions) is less pronounced and much smaller than in the case of the lighter negative ions.

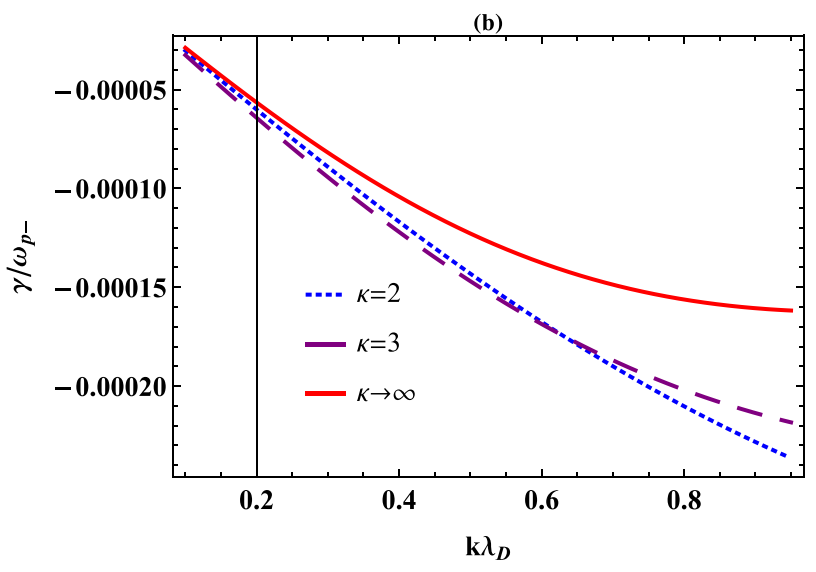

FIG. 3. Normalized damping rates $\gamma / \omega_{p-}$ as a function of the normalized wave number $k \lambda_{D}$ for IAWs propagating in a non-thermal dust bi-ion electron plasma. The damping rates are plotted for several spectral indices $\kappa$. (a) illustrates the case of the $K^{+}-S F_{6}^{-}$DBIE mixture, while (b) shows the damping rate for the heavier $K^{+}-C_{7} F_{14}^{-}$mixture. 

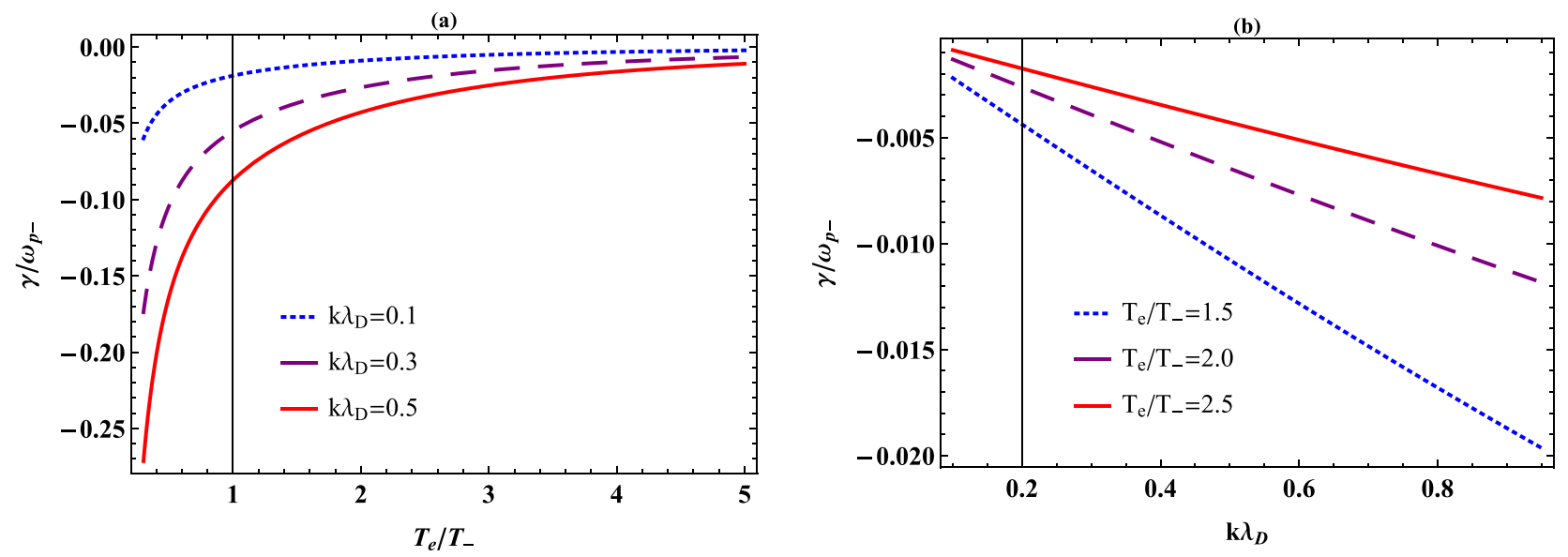

FIG. 4. Normalized damping rates $\gamma / \omega_{p-}$ vs. temperature ratios (a) and normalized wave number $k \lambda_{D}$ (b) for IAW in a non-thermal dust bi-ion $\left(K^{+}-S F_{6}^{-}\right)$ electron plasma.

Figure 4 displays the variation of the normalized damping rates for modified IAWs in a kappa-distributed Potassium-Sulphur Hexafluoride $\left(K^{+}-S F_{6}^{-}\right)$electron plasma in the presence of stationary dust. Fig. 4(a) shows the dependence of the damping rate on the temperature ratio between the electrons and the negative ions at several fixed wavelengths. The temperature ratio is set to $T_{e} / T_{-}=2.5$, and the spectral index for this case is set to $\kappa=2$. Fig. 4(b) shows the dispersion of the IAWs for a selected set of temperature ratios. The wavelength in all plots is normalized to the Debye length $\lambda_{D}$.

Figure 5 presents contour plots showing the variation of the IAW damping rates. Fig. 5(a) shows the dependence on the temperature ratio (electrons to negative ions) and the normalized wave number. Fig. 5(b) illustrates the dependence on the temperature ratio and the product of dust particles' number density (relative to the number density of positive ions) $\eta \equiv n_{d} / n_{0+}$ and the dust particles' charge potential $Z_{D}$. The spectral index for both plots is set to $\kappa=2$. The product of the number density and the charge potential in the upper plot is set to $\eta Z_{D}=0.3$. The wavelength in the bottom panel is set to $k \lambda_{D}=0.1$. The results show that the damping of the IAWs increases with decreasing temperature ratios. Simultaneously, the damping increases with the increasing wave number but remains almost unaffected by the charging potential or the relative dust particles' number density.

Figure 6 illustrates the variation of the normalized damping rates of modified IAWs propagating in a kappa-distributed Potassium-Perfluoromethylcyclohexane $\left(K^{+}-C_{7} F_{14}^{-}\right)$DBIE plasma. Similar to Fig. 4, Fig. 6(a) shows the dependence on the temperature ratios at fixed wavelengths, while Fig. 6(b) shows the dependence on the normalized wave number at fixed temperature ratios. The damping rate shows similar behavior to that for the $K^{+}-S F_{6}^{-}$mixture. Yet, the IAWs in the case of the $K^{+}-C_{7} F_{14}^{-}$DBIE plasma are less damped. We should note that for temperature ratios $T_{e} / T_{-}<1$, the model assumptions are not fulfilled (due to the restriction in the Lorentzian thermal velocities, see Sec. II); therefore, the damping values below this limit should not be considered. The spectral index in this case is set to $\kappa=2$.

Figure 7 presents similar contour plots of the IAW damping rate to the ones described in Fig. 5 but applied to the mixture with heavier negative ions, $K^{+}-C_{7} F_{14}^{-}$. Fig. 7(a) shows the variation of the damping rate with respect to the temperature ratio and the normalized wave number. Fig. 7(b) shows the combined gradual dependence of the damping rate on the temperature ratio and the product of the
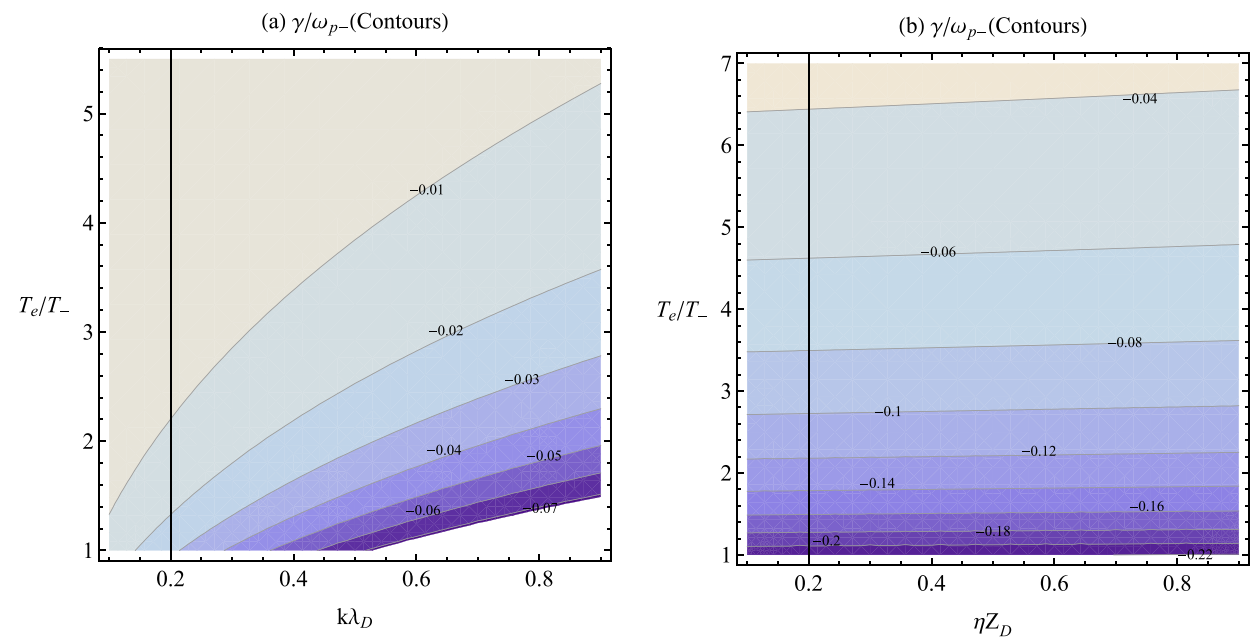

FIG. 5. Contour plots of the damping rate of the IAWs in the case of the $K^{+}-S F_{6}^{-}$ion mixture. (a) shows the variation with respect to the electronsto-negative ions' temperature ratio and the normalized wave number $k \lambda_{D}$. (b) illustrates the slow variation of the damping rate with the temperature ratio and the product of dust particles' number density relative to the number density of positive ions $\eta$ and the dust particles' charge potential $Z_{D}$. 
(a)

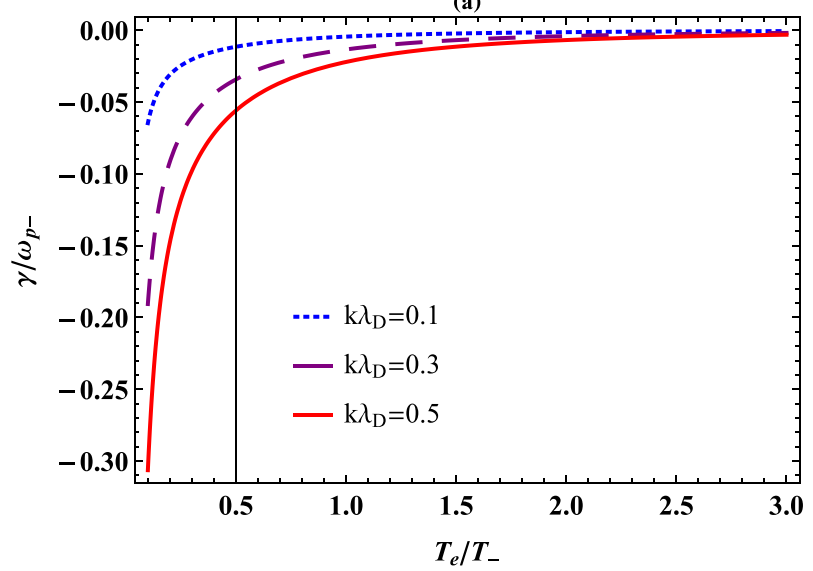

(b)

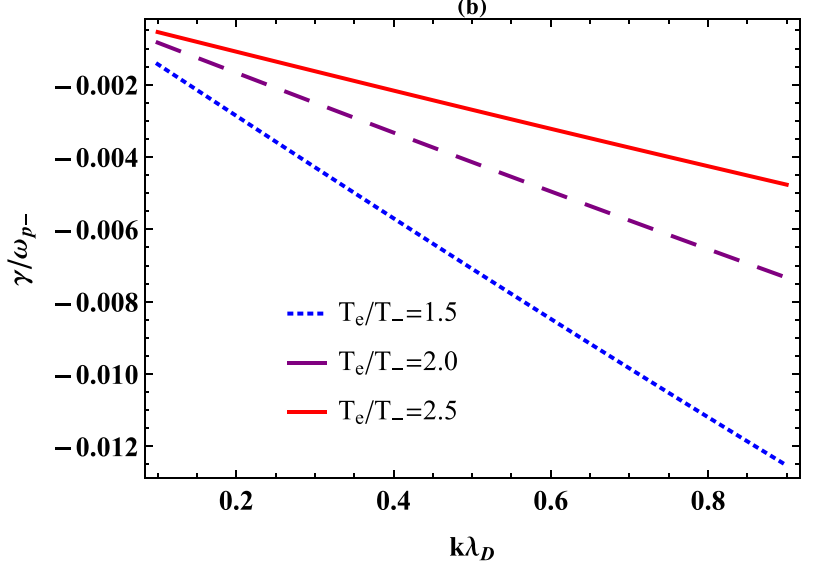

FIG. 6. Normalized damping rates $\gamma / \omega_{p-}$ vs. temperature ratios (a) and normalized wave number $k \lambda_{D}$ (b) for the case of non-thermal dust bi-ion $K^{+}-C_{7} F_{14}^{-}$ electron plasma.

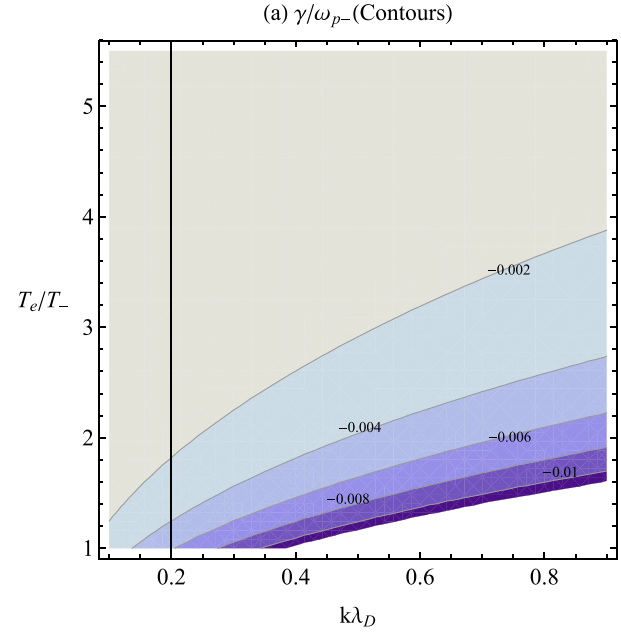

relative number density $\eta$ and the charge potential for the dust particles. Similar to the case of the Sulphur Hexafluoride ion mixture, the damping rate here increases with the decreasing temperature ratio and increasing wave number and charging potential and/or relative number density for the dust ions. The product of the number density and the charge potential for Fig. 7 (a) is set to $\eta Z_{D}=0.3$, while the wave number in Fig. 7(b) is set to $k \lambda_{D}=0.1$ as above. Whenever not explicitly specified in the remaining plots, the

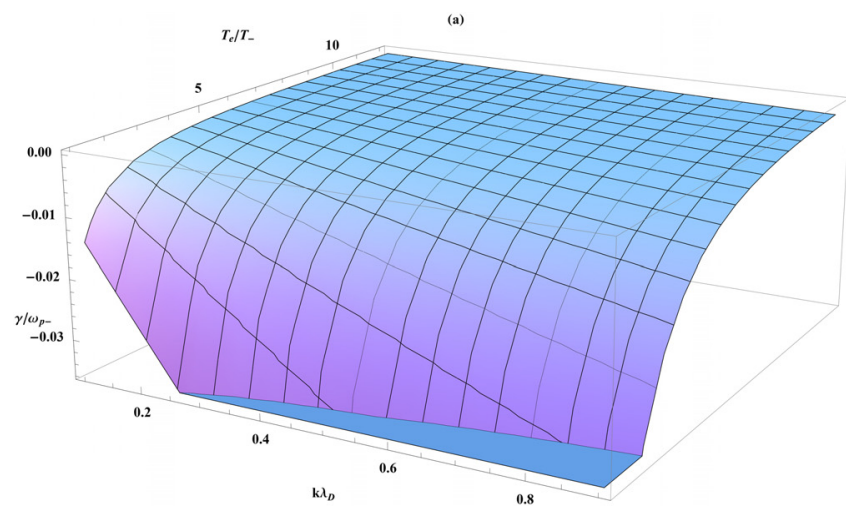

(b) $\gamma / \omega_{p-}$ (Contours)

FIG. 7. Contour plots of the damping rate of the IAWs in the case of $K^{+}-$ $C_{7} F_{14}^{-}$ions. (a) shows the variation with respect to the electrons-to-negative ions' temperature ratio and the normalized wave number $k \lambda_{D}$. (b) illustrates the gradual variation of the collisionless Landau damping with the temperature ratio and the product of dust particles' number density relative to the number density of positive ions $\eta$ and the dust particles' charge potential $Z_{D}$.

FIG. 8. A 2D surface plot of the estimated kinetic damping rate in the lighter $K^{+}-S F_{6}^{-}$DBIE mixture. (a) shows the dependence on the electron-to-negative ions' temperature ratio and the product of the dust charging potential and the relative density. (b) illustrates the damping as a function of the temperature ratio and the normalized wave number.

plasma parameters are set to $\kappa=2, \eta Z_{D}=0.3$, and $k \lambda_{D}=0.1$.

To further illustrate the dependence of the estimated IAW damping rate on the various plasma properties, we have created separate surface plots for each ion mixture, as presented in the following figures.

Figure 8 shows a surface plot of the estimated damping rate in the case of the lighter $K^{+}-S F_{6}^{-}$DBIE mixture. Fig. 8(a) shows the dependence on the electron-to-negative ions'

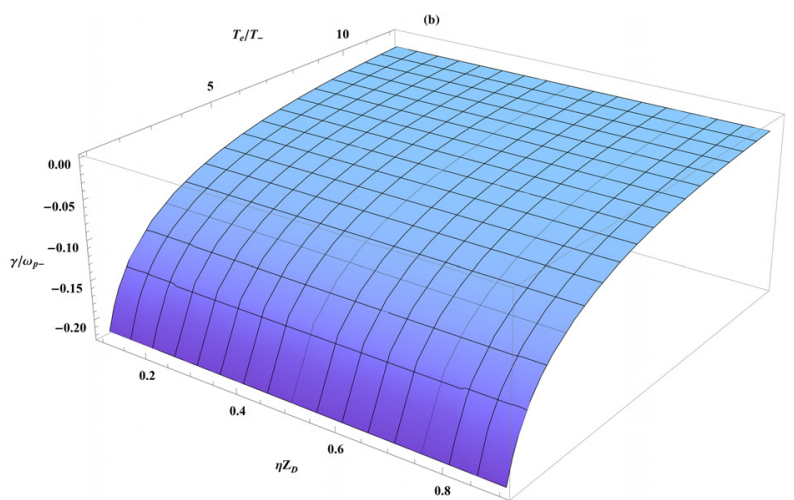



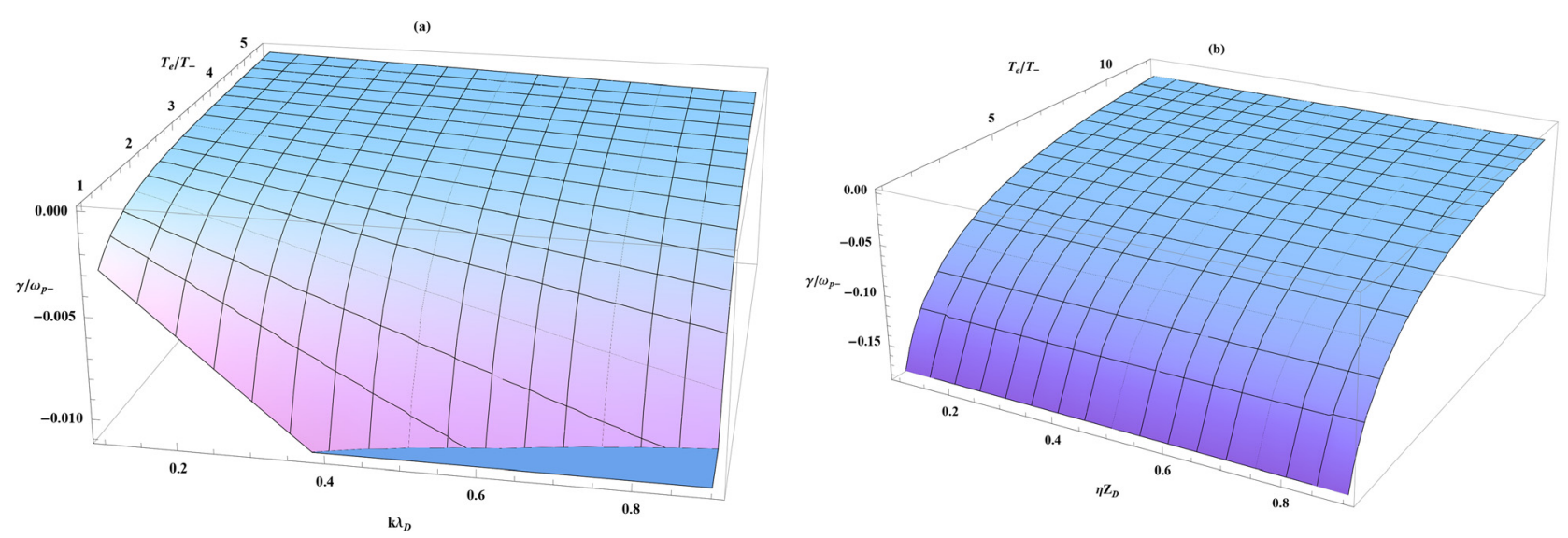

FIG. 9. Surface plots of the estimated damping rate in the case of heavier $K^{+}-C_{7} F_{14}^{-}$DBIE mixture. (a) demonstrates the damping as a function of the temperature ratio and the product of the dust charging potential and the relative density. (b) illustrates the dependence on the temperature ratio and the normalized wave number.

temperature ratio and the product of the dust charging potential and the relative density. Fig. 8(b) illustrates the damping rate as a function of the temperature ratio and the normalized wave number at fixed wavelength $k \lambda_{D}=0.1$ and fixed spectral index $\kappa=2$. Once again, we observe the same behavior for the damping rate as the one discussed in Fig. 5.

Figure 9 shows a surface plot of the estimated damping rate in the case of the heavier $K^{+}-C_{7} F_{14}^{-}$DBIE mixture. Similar to Fig. 8, Fig. 9(a) shows the dependence on the electron-to-negative ions' temperature ratio and the product of the dust charging potential and the relative density. Fig. 9(b) illustrates the damping rate as a function of the temperature ratio and the normalized wave number at fixed wavelength $k \lambda_{D}=0.1$ and fixed spectral index $\kappa=2$.

\section{SUMMARY AND CONCLUSIONS}

To summarize, we have estimated the Landau damping rates within the weak damping approximation in nonMaxwellian dust bi-ion electron plasmas comprising electrons, positive-negative ions, and stationary negative dust particles. We have studied two separate mixtures consisting of different negatively charged ions, namely, Potassium-Sulphur Hexafluoride $K^{+}-S F_{6}^{-}$and PotassiumPerfluoromethylcyclohexane $K^{+}-C_{7} F_{14}^{-}$. Our approach takes into account supra-thermal Lorentzian distributed plasmas and discusses the role of various spectral indices $\kappa \geq 2$. We should note that the dominant collisional damping is not taken into consideration here. Including it will lead to a modification of the dielectric tensor of the respective mode. Our results are mainly based on a fixed charging potential of the dust particles, where the particle collisions are weak due to a lower dust-particle collision frequency. In this respect, we focus on the role of the non-thermal kinetic effects for the IAW damping in the Lorentzian multi-species charged plasmas including the effect of negative ions, which has been previously underlooked in the literature.

When we compare the estimated damping rates for Maxwellian and non-thermal plasmas, we find weaker Landau damping in the case of Maxwellian plasma (smaller magnitude or absolute value of $\left.\gamma / \omega_{p-}\right)$ and stronger damping in the case of non-thermal Lorentzian plasmas (larger magnitude or absolute value of $\gamma / \omega_{p-}$ ) due to the increase in the number of energetic particles in the tail of the velocity distribution function. It is natural that when a larger number of particles take energy from a given IAW, the damping of the wave will increase. While the damping is enhanced, the real frequency in the case of non-thermal plasmas decreases and the phase speed of the IAWs slows down as we transition from the thermal Maxwellian to Lorentzian case. This conclusion is based on the analysis of the frequency and damping rate variations for several spectral indices, varying from highly supra-thermal distributions with $\kappa=2$ to regular Maxwellians with $\kappa \rightarrow \infty$.

Furthermore, we have investigated the dispersion and the influence of other plasma parameters, such as the temperature ratio between the electrons and the negatively charged ions, the relative dust number density, and the charging potential of the dust ions. The results clearly show that with the increasing electron-ion temperature ratio, the damping rate $\gamma / \omega_{p-}$ of the dust bi-ion electron plasma decreases. Next, we consider the impact of the number density of the stationary dust particles and the negative ions and find that within the selected range, they do not play a significant role in the damping of the IAW. Yet, the inertia of the negative ions plays a role in the attenuation of the waves and the IAWs are damped quicker in the case of mixtures with heavy negative ions.

We also expect that at very large inertia of the dust particles, the waves will be damped and their excitation would be hindered.

To conclude, we have discussed the Landau damping and the possible unstable regimes of growing modified IAWs in a non-Maxwellian dust bi-ion electron plasma. Previous studies on the subject focus mainly on the damping rates in the presence of pure positive ions. In our study, we have stressed on the kinetic effects and the modification of the damping rates in the presence of different negative ions. Laboratory experiments ${ }^{40}$ have shown that in the simultaneous presence of relatively cold electrons and positive ions, colder and much heavier negative ions, and a separate population of dust particles, the dust particles might change their potential and a transition from negative to positive charging of the dust particles becomes possible. According to our model at a fixed 
wave-number, temperature ratio, and Lorentzian index $\kappa$, the damping rate of the IAWs within such a multi-species plasma mixture is weakly affected by the product of the dust particles' number density and their charging potential. Our results indicate that the Landau damping is most affected by the temperature ratio between the electrons and the negative ions, which can lead to an order of magnitude difference in the damping rate. The dispersion effects (the wave-number dependence) are of secondary importance, followed by the variation in the Lorentzian index $\kappa$. Since collisional damping has been neglected, the focus of our paper is on the kinetic effects and the role of collisionless Landau damping as a function of the various plasma parameters as discussed above. The dust particles in our model are assumed to be orders of magnitude heavier than all the ion species, and they are considered to be stationary. Depending on the collisional cross-sections, for fixed temperatures and number density of the dust particles, we expect the effect of the dust-ion collisions to remain the same for all values of the spectral $\kappa$, as well as the charging potential parameter $\eta Z_{D}$. In this respect, we can presume that the role of the molecular collisions would remain the same within the conditions considered within our paper while the kinetic damping has stronger dependence on the plasma parameters. Our findings can significantly contribute to the current understanding of the IAW properties in laboratory settings ${ }^{28}$ and dusty astrophysical environment, such as cometary tails, ${ }^{55,56}$ circumstellar environment, and presolar nebula. ${ }^{57,58}$

\section{ACKNOWLEDGMENTS}

Y.M. would like to acknowledge the support from FWO-Research Foundation Flanders: Postdoctoral Fellowship Grant No. 12K1416N. The work had also received funding from projects C 90347 (ESA Prodex), GOA/2015014 (KU Leuven) and G.0A23.16N (FWO-Vlaanderen).

${ }^{1}$ N. Krall and A. Trivelpiece, Principles of Plasma Physics (McGraw-Hill, New York, 1973).

${ }^{2}$ L. Tonks and I. Langmuir, Phys. Rev. 33, 195 (1929).

${ }^{3}$ R. W. Revans, Phys. Rev. 44, 798 (1933).

${ }^{4}$ A. Y. Wong, R. W. Motley, and N. D. Angelo, Phys. Rev. A 133, 436 (1964).

${ }^{5}$ F. Verheest, Waves in Dusty Space Plasmas (Kluwer Academic, Dordrecht, 2000).

${ }^{6} \mathrm{P}$. K. Shukla and A. A. Mamun, Introduction to Dusty Plasma Physics (Institute of Physics, Bristol, 2002).

${ }^{7}$ T. J. Millar, C. Walsh, M. A. Cordiner, R. N. Chuimn, and E. Herbst, Astrophys. J. 662, L87 (2007).

${ }^{8}$ J. Cernicharo, Spectrosc. Space 20, 23 (2001).

${ }^{9}$ C. J. Mitchell and M. Horanyi, Science 311, 1587 (2006).

${ }^{10}$ U. de Angelis, V. Formisano, and M. Giordano, J. Plasma Phys. 40, 399 (1988).

${ }^{11}$ V. V. Yaroshenko, F. Verheest, and G. E. Morfill, Astron. Astrophys. 461, 385 (2007).

${ }^{12}$ S. I. Kopnin, S. I. Popel, and M. Y. Yu, Phys. Plasmas 16, 063705 (2009).

${ }^{13}$ G. E. Morfill and A. V. Ivlev, Rev. Mod. Phys. 81, 1353 (2009).

${ }^{14}$ R. L. Merlino, in Plasma Physics Applied, edited by C. Grabbe (Transworld Research Network, Kerala, 2006).

${ }^{15}$ V. E. Fortov, A. V. Ivlev, S. A. Khrapak, A. G. Khrapak, and G. E. Morfill, Phys. Rep. 421, 1 (2005).

${ }^{16}$ N. N. Rao, P. K. Shukla, and M. Yu, Planet. Space Sci. 38, 543 (1990); P. K. Shukla and V. P. Silin, Phys. Scr. 45, 508 (1992).

${ }^{17}$ W. M. Moslem, R. Sabry, S. K. El-Labany, and P. K. Shukla, Phys. Rev. E 84, 066402 (2011).
${ }^{18}$ M. M. Masud, M. Asaduzzaman, and A. A. Mamun, Astrophys. Space Sci. 343, 221 (2013); J. Plasma Phys. 79, 215 (2013).

${ }^{19}$ T. Trottenberg, D. Block, and A. Piel, Phys. Plasmas 13, 042105 (2006).

${ }^{20}$ M. Rosenberg and R. L. Merlino, J. Plasma Phys. 75, 495 (2009).

${ }^{21}$ M. Rosenberg and R. L. Merlino, Planet. Space Sci. 55, 1464 (2007).

${ }^{22}$ R. L. Merlino, A. Barkan, C. Thompson, and N. D’Angelo, Phys. Plasmas 5, 1607 (1998).

${ }^{23}$ E. Thomas, R. Fisher, and R. L. Merlino, Phys. Plasmas 14, 123701 (2007).

${ }^{24}$ M. Rosenberg, E. Thomas, and R. L. Merlino, Phys. Plasmas 15, 073701 (2008).

${ }^{25}$ R. L. Merlino, Phys. Plasmas 16, 124501 (2009).

${ }^{26}$ R. L. Merlino and S.-H. Kim, J. Chem. Phys. 129, 224310 (2008).

${ }^{27}$ J. Vranjes, Phys. Plasmas 18, 084501 (2011).

${ }^{28}$ S.-H. Kim and R. L. Merlino, Phys. Rev. E 76, 035401 (2007).

${ }^{29}$ A. A. Mamun, R. A. Cairns, and P. K. Shukla, Phys. Lett. A 373, 2355 (2009).

${ }^{30}$ T. Chapman, R. L. Berger, S. Brunner, and E. A. Williams, Phys. Rev, Lett. 110, 195004 (2013).

${ }^{31}$ F. Melandso, T. Aslaksen, and O. Havnes, Planet. Space Sci. 41, 321 (1993).

${ }^{32}$ F. Melamdso, T. K. Aslaksen, and O. Havnes, J. Geophys. Res. 98, 315 (1993).

${ }^{33}$ S. Ali, Phys. Plasmas 16, 113706 (2009).

${ }^{34}$ V. M. Vasyliunas, J. Geophys. Res. 73, 2839, doi:10.1029/ JA073i009p02839 (1968).

${ }^{35}$ R. M. Thorne and D. Summers, Phys. Fluids B 3, 2117 (1991).

${ }^{36}$ Z. Meng, R. M. Thorne, and D. Summers, J. Plasma Phys. 47, 445 (1992).

${ }^{37}$ R. L. Mace, M. A. Hellberg, and R. A. Treumann, J. Plasma Phys. 59, 393-416 (1998).

${ }^{38}$ M. A. Hellberg and R. L. Mace, Phys. Plasmas 9, 1495-1504 (2002).

${ }^{39}$ M. Hellberg, R. Mace, and T. Cattaert, Space Sci. Rev. 121, 127 (2005).

${ }^{40}$ N. S. Saini, I. Kourakis, and M. A. Hellberg, Phys. Plasmas 16, 062903 (2009).

${ }^{41}$ S. Sultana, I. Kourakis, N. S. Saini, and M. A. Hellberg, Phys. Plasmas 17, 032310 (2010).

${ }^{42}$ T. K. Baluku, M. A. Hellberg, N. S. Saini, and I. Kourakis, Phys. Plasmas 17, 053702 (2010).

${ }^{43}$ T. K. Baluku and M. A. Hellberg, Phys. Plasmas 19, 012106 (2012).

${ }^{44}$ F. Verheest, M. A. Hellberg, and I. Kourakis, Phys. Plasmas 20, 082309 (2013).

${ }^{45}$ M. A. Hellberg, R. L. Mace, T. K. Baluku, I. Kourakis, and N. S. Saini, Phys. Plasmas 16, 094701 (2009).

${ }^{46}$ A. Danehkar, N. S. Saini, M. A. Hellberg, and I. Kourakis, Phys. Plasmas 18, 072902 (2011).

${ }^{47}$ E. I. El-Awady, S. A. Altantawy, W. M. Moslem, and P. K. Shukla, Phys. Lett. A 374, 3216 (2010)

${ }^{48}$ Z. Liu and J. Du, Phys. Plasmas 16, 123707 (2009); K. Arshad, S. Mahmood, and A. M. Mirza, ibid. 18, 092115 (2011); K. Arshad and S. Mahmood, ibid. 17, 124501 (2010); G. Livadiotis, JGR 120, 1607 (2015).

${ }^{49}$ K. Arshad, A. Rehman, and S. Mahmood, Phys. Plasmas 22, 112114 (2015); 23, 052107 (2016).

${ }^{50}$ K. Arshad and A. M. Mirza, Astrophys. Space Sci. 349, 753 (2014); K. Arshad, F. Siddique, A. M. Mirza, and A. Rehman, ibid. 350, 169 (2014); $\mathbf{5 8 5}, 169$ (2014)

${ }^{51}$ R. L. Mace and M. A. Hellberg, Phys. Plasmas 16, 072113 (2009); K. Arshad, Z. Ehsan, S. A. Khan, and S. Mahmood, ibid. 21, 023704 (2014); G. Livadiotis and D. J. McComas, Space Sci. Rev. 175, 183 (2013); I. Kourakis, S. Sultana, and M. A. Hellberg, Plasma Phys. Controlled Fusion 54, 124001 (2012); K. Arshad, M. Lazar, S. Mahmood, A. Rehman, and S. Poedts, Phys. Plasmas 24, 033701 (2017); M. Lazar, S. Poedts, and R. Schlickeiser, Astron. Astrophys. 534, A116 (2011).

${ }^{52}$ P. Tolias, S. Ratynskaia, and U. de Angelis, Phys. Plasmas 17, 103707 (2010).

${ }^{53}$ V. N. Tsytovich and U. de Angelis, Phys. Plasmas 6, 1093 (1999); 7, 554 (2000); 8, 1141 (2001).

${ }^{54}$ D. A. Gurnett and A. Bhattacharjee, Introduction to Plasma Physics (Cambridge University Press, 2005).

${ }^{55}$ N. D. Angelo and B. Song, Planet. Space Sci. 38, 1577 (1990).

${ }^{56}$ H. Gunell, H. Nilsson, M. Hamrin, A. Eriksson, E. Odelstad, R. Maggiolo, P. Henri, X. Vallieres, K. Altwegg, C.-Y. Tzou, M. Rubin, K.-H. Glassmeier, G. Stenberg Wieser, C. Simon Wedlund, J. De Keyser, F. Dhooghe, G. Cessateur, and A. Gibbons, Astron. Astrophys. 600, A3 (2017).

${ }^{57}$ M. Horanyi and C. K. Goertz, Astrophys. J. 361, 155 (1990).

${ }^{58}$ D. A. Mendis and M. Rosenberg, Annu. Rev. Astron. Astrophys. 32 419-463 (1994).

${ }^{59}$ L. D. Landau, Sov. Phys. JETP 10, 25 (1946). 